\title{
An investigation into natural vibrations of fluid-structure interaction systems subject to Sommerfeld radiation condition
}

\author{
Jing Tang Xing
}

Received: 1 March 2007 / Revised: 28 September 2007 / Accepted: 11 October 2007 / Published online: 10 January 2008

(C) Springer-Verlag 2008

\begin{abstract}
A fluid-structure interaction system subject to Sommerfeld's condition is defined as a Sommerfeld system which is divided into three categories: Fluid Sommerfeld (FS) System, Solid Sommerfeld (SS) System and Fluid Solid Sommerfeld (FSS) System of which Sommerfeld conditions are imposed on a fluid boundary only, a solid boundary only and both fluid and solid boundaries, respectively. This paper follows the previous initial results claimed by simple examples to further mathematically investigate the natural vibrations of generalized Sommerfeld systems. A new parameter representing the speed of radiation wave for generalized 3-D problems with more complicated boundary conditions is introduced into the Sommerfeld condition which allows investigation of the natural vibrations of a Sommerfeld system involving both free surface and compressible waves. The mathematical demonstrations and selected examples confirm and reveal the natural behaviour of generalized Sommerfeld systems defined above. These generalized conclusions can be used in theoretical or engineering analysis of the vibrations of various Sommerfeld systems in engineering.
\end{abstract}

Keywords Sommerfeld system - Complex natural frequencies · Fluid-structure interaction - Complex energy flow identity $\cdot$ Speed of radiation wave

\section{Introduction}

Sommerfeld's original proof [1] of the uniqueness theorem of the radiation solution $\varphi=\phi \mathrm{e}^{-i \omega t}$ of the wave equation defined in a full infinite 3-dimensional space assumed an

\section{J. T. Xing $(\varangle)$}

School of Engineering Sciences, University of Southampton,

Southampton SO17 1BJ, England

e-mail: jtxing@soton.ac.uk additional condition

$\lim _{r \rightarrow \infty} r\left(\frac{\partial \phi}{\partial r}-i \kappa \phi\right)=0, \quad \kappa=\omega / c$.

Here, the quantity $r$ stands for the distance from any fixed point in the space $r=0, i=\sqrt{-1}, \kappa$ represents the ratio of the circular frequency $\omega$ of the stimulation and $c$ the speed of wave in a full infinite three space. This condition is called the general condition of radiation [2]. The fact that this condition is superfluous has been rigorously proven by Rellich [3] even for the case of an arbitrary number of dimensions $h$ where the radiation condition reads

$\lim _{r \rightarrow \infty} r^{\frac{h-1}{2}}\left(\frac{\partial \phi}{\partial r}-i \kappa \phi\right)=0$.

Courant and Hilbert [4] further discussed and demonstrated the characterisation of outward energy radiation of wave radiation problems satisfying the Sommerfeld condition in the form given by Magnus and Oberhettinger [5]

$$
\lim _{r \rightarrow \infty} \int_{r=\left|x-x^{\prime}\right|}\left|\left(\frac{\partial \phi}{\partial r}-i \kappa \phi\right)\right|^{2} \mathrm{~d} S_{r}=0,
$$

where $\mathrm{d} S_{r}$ denotes the surface element of a large sphere $S_{r}$ of radius $r$ about a fixed point $x^{\prime}$. As mentioned by Courant and Hilbert [4], this integration form is less demanding than Eq. (2), yet it suffices for the characterisation of outward radiation.

For a problem not defined in a full infinite space, finite boundary conditions are involved, such as the example investigated in Sect. 7.4. The speed of the radiation wave may be different from the speed of the pressure wave or surface wave. Therefore, we choose notations $k$ and $\xi$, depending on the problem, to represent the integrated speed of wave in the radiation condition. The variable $\xi$ is a positive real number 
to satisfy the case of $\xi=c$ for a full infinite domain problem discussed by Sommerfeld. The radiation condition is written in a general form as

$$
\begin{aligned}
& \lim _{r \rightarrow \infty} r^{\frac{h-1}{2}}\left(\frac{\partial \phi}{\partial r}-i k \phi\right)=0, \\
& k=\frac{\omega}{\xi} \\
& \varphi=\phi \mathrm{e}^{-i \omega t} .
\end{aligned}
$$

Considering natural vibrations $\varphi=\phi \mathrm{e}^{-i \omega t}$, Eq. (4) is written in an equivalent form.

$$
\lim _{r \rightarrow \infty} r^{\frac{h-1}{2}}\left(\frac{\partial \varphi}{\partial r}+\frac{1}{\xi} \frac{\partial \varphi}{\partial t}\right)=0
$$

Sommerfeld's condition physically represents the case that waves produced and radiated from the sources must scatter to infinity with the velocity $c$ or $\xi$ in $r$ direction and no waves may be radiated from infinity into the prescribed singularities of the field. Similarly, for a wave coming from infinity, the absorption condition defined by Sommerfeld [2] has a form for an arbitrary number of dimensions $h$

$\lim _{r \rightarrow \infty} r^{\frac{h-1}{2}}\left(\frac{\partial \phi}{\partial r}+i k \phi\right)=0$.

Sommerfeld's condition has been widely adopted to investigate incompressible water-wave radiation problems, for example see, Newman [6], Bishop and Price [7], Eatock Taylor [8].

In the acoustic field, Gaunaurd and Brill [9] presented a study of the resonance scattering problem of an infinitelong axisymmetric elastic cylinder excited by an incident plane wave. The far-field water pressure was expressed by the Hankel function to manage a wave condition at an infinite boundary. The characteristic matrix of the system was presented. In a comprehensive critical review paper by Tang and Fan [10] the mechanisms of sound scattering and radiation of submerged elastic structure-acoustic interaction system excited by incident wave was further discussed. In the conclusion, it is clearly mentioned that the roots of the characteristic equation of the studied structure-acoustic coupling system are complex. Moreover, Filippi [11] used a Fourier transformation method to derive the dynamic response of a one-dimensional vibro-acoustic system excited by an external force and subject to the imposed Sommerfeld's radiation condition at infinity. However, the main objective in the above publications mentioned was to study the dynamic responses of the system excited by external excitations or vibration sources but it was not intended to explore the natural behaviours of the system in which there are no external excitations applied, although the characteristic equation of the individual studied system was involved [9-11].

As is well known (see, for example $[4,12]$ ), the natural vibration of a dynamic system is defined by an eigenvalue problem of the corresponding idealised system with no material damping assumed and no external forces. From the defined eigenvalue problem, the real natural frequencies and modes of the system are derived or calculated by using finite element methods $[13,14]$. For example Morand and Ohayon [15] presented some detailed methods for numerical modelling of linear natural vibration analysis of elastic structures coupled to internal fluids. Xing and Price [16] as well as Xing et al. [17] proposed a mixed finite element substructuresubdomain method to simulate natural vibrations and dynamic responses of various linear fluid-structure interaction problems.

A fluid-structure interaction system subject to a Sommerfeld's condition is defined as a Sommerfeld system in this paper. Following the definition of natural vibration of a system, we may ask a question: what are the natural dynamic characteristics of a Sommerfeld system? Here it is needed to clarify again that in this natural vibration system studied in this paper, there are no excitations, such as incident waves, vibration sources etc. applied. The main aims of this research are to reveal the natural characteristics of the system. These characteristics are independent of any external excitations of the system.

To address this problem, Xing et al. [18] investigated the natural vibrations of beam-water interaction systems. In this paper, the natural vibration of the beam-water interaction system under an undisturbed condition at the infinite boundary of the water was well solved but it was unsuccessful in solving the case of the system with a Sommerfeld condition, although it was mentioned that "there exists no real solution for the eigenvalue equation under the Sommerfeld condition". To confirm this by an example, Xing (communication note $\mathrm{L} / 28 / 02$, ship science, University of Southampton, 2002) solved the natural vibration of a linear 1-D fluidstructure interaction system (Fig. 2) consisting of a massspring system coupled to an infinite fluid domain subject to a Sommerfeld radiation condition at an infinite boundary. It was found that the natural vibration of this simple system is governed by a complex eigenvalue problem and its complex frequency was obtained. This note was cited by Zhao [19] who followed the idea and used the method by Xing et al. [18] to calculate the natural frequencies of a beam-water interaction system subject to a Sommerfeld condition at an infinite fluid boundary. For the case with no free surface wave, the complex frequency of the system was obtained [19]. Unfortunately, it failed to find any solution of the natural vibration of the system with a free surface wave considered. Later, Xing [20] extended the one mass-spring system to a two massesspring system shown in Fig. 3, which increased the degree 
of freedom of the solid from one to two, to solve its natural vibration. The two conjugate complex eigenvalues were obtained.

All of these mentioned results, including the discussion in Refs. $[9,10]$, are obtained only by individual simple examples. Therefore, the solution of the natural vibrations of a generalized Sommerfeld system has not been reported. Moreover, it is not known the fail reason [19] for the case of the beam-water Sommerfeld system including a free surface wave condition on the water surface. This paper aims to investigate this fundamental problem from a generalized mathematical model covering the various Sommerfeld systems in engineering. Based on this generalized investigation, the previously obtained individual conclusions are further confirmed and extended to the generalized cases. Furthermore, a new parameter representing the speed of radiation wave in complex boundary conditions is introduced to answer the fail reason by Zhao [19].

\section{Definitions}

A fluid-structure interaction system is defined as a Sommerfeld fluid-structure interaction system if its boundary conditions include at least a Sommerfeld radiation condition in Sect. 1 or 3. A Sommerfeld fluid-structure interaction system may have a Sommerfeld radiation condition on its solid boundary, or fluid boundary or both fluid and solid boundaries. Depending on the location of the Sommerfeld radiation condition only on the fluid boundary, or only on the solid boundary or on both of the fluid and solid boundaries of the system, we call a Sommerfeld fluid-structure interaction system as a fluid-Sommerfeld system (FS System), or a solid-Sommerfeld system (SS System), or a fluid-solidSommerfeld system (FSS System), respectively.

\section{Governing equations}

An FS System is shown in Fig. 1. The solid is a flexible structure of mass density $\rho_{s}$ in a domain $\Omega_{s}$ of boundary $S=S_{u} \cup S_{T} \cup S_{w} \cup \Sigma$ with a unit normal vector $v_{i}$ pointing outside. The fluid of mass density $\rho_{f}$ in a domain $\Omega_{f}$ of boundary $\Gamma=\Gamma_{f} \cup \Gamma_{b} \cup \Sigma \cup \Gamma_{\infty}$ with a unit outside normal vector $\eta_{i}$ is assumed compressible, inviscous and its motion irrotational. A fixed coordinate system $o-x_{1} x_{2} x_{3}$ is chosen to investigate small motions of the system, so that the coordinate system is considered as an Eulerian system for the fluid and also a material system for the solid. The Cartesian tensors [21] with subscripts $i, j, k$ and $l$, etc. $(=1,2,3)$ obeying the summation convention are used. For example $U_{i}, V_{i}, e_{i j}$ and $\sigma_{i j}$ represent displacement and velocity vectors, strain and stress tensors of the elastic solid, respectively; $p, v_{i}$ and

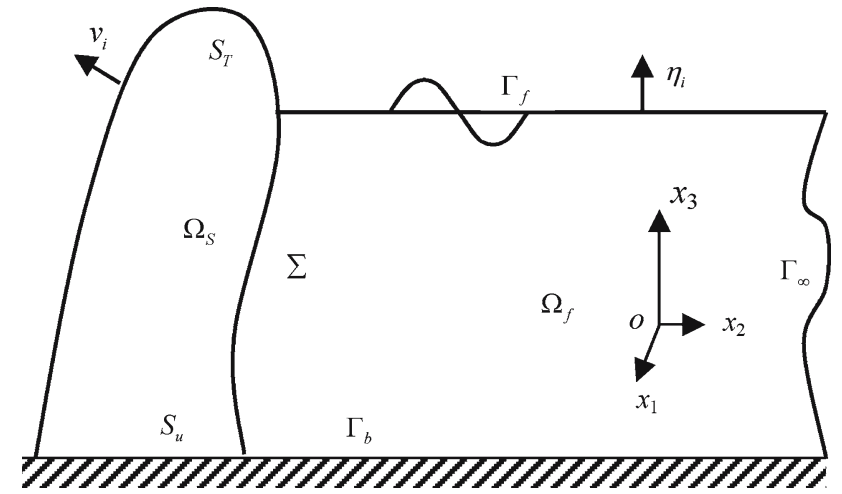

Fig. 1 A fluid-Sommerfeld fluid-structure interaction system (FS System)

$\varphi$, respectively, denote the dynamic pressure, velocity and the velocity potential of the fluid. Notations ()$_{, t}=\partial() / \partial t$, $U_{i, j}=\partial U_{i} / \partial x_{j}, V_{i}=U_{i, t}=\partial U_{i} / \partial t$, Kronecker delta $\delta_{i j}$ and permutation tensor $e_{i j k}$ are adopted. The elastic tensor of the solid material and the speed of sound in the fluid are represented by $E_{i j k l}$ and $c$, respectively. According to the linear theory, the governing equations describing the natural vibration of this system are as follows.

\subsection{Solid structure}

Dynamic equation

$\sigma_{i j, j}=\rho_{s} V_{i, t}, \quad\left(x_{i}, t\right) \in \Omega_{s} \times\left(t_{1}, t_{2}\right)$.

Geometric relationships

$e_{i j}=\left(U_{i, j}+U_{j, i}\right) / 2$

$V_{i}=U_{i, t}, \quad\left(x_{i}, t\right) \in \Omega_{s} \times\left(t_{1}, t_{2}\right)$.

Constitutive equation

$\sigma_{i j}=E_{i j k l} e_{k l}, \quad\left(x_{i}, t\right) \in \Omega_{s} \times\left(t_{1}, t_{2}\right)$.

For isotropic materials, the tensor $E_{i j k l}$ has the following form [21]

$E_{i j k l}=[2 G v /(1-2 v)] \delta_{i j} \delta_{k l}+G\left(\delta_{i k} \delta_{j l}+\delta_{i l} \delta_{j k}\right)$,

where $G$ and $v$ denote shear modulus and Poisson's ratio of the material, respectively.

Boundary conditions

displacement:

$U_{i}=o, \quad\left(x_{i}, t\right) \in S_{u} \times\left[t_{1}, t_{2}\right]$,

traction:

$\sigma_{i j} v_{j}=0, \quad\left(x_{i}, t\right) \in S_{T} \times\left[t_{1}, t_{2}\right]$. 


\section{Navier's equation}

Substituting Eqs. (8)-(11) into (7), we obtain the Navier's equation

$G\left[U_{i, j j}+1 /(1-2 v) U_{j, j i}\right]=\rho_{s} U_{i, t t}$.

A general radiation condition in the $x_{i}$ direction for this equation is defined as

$\lim _{x_{j} \rightarrow \infty}\left(\tilde{U}_{i, j} v_{j}-i k \tilde{U}_{i}\right)=0$,

where $U_{i}=\tilde{U}_{i} \mathrm{e}^{-i \omega t}$. To demonstrate that these two Eqs. (14) and (15) cover general cases, we can derive the equations for volume (or pressure) waves and shear waves as follows. Taking a divergence operation on Eqs. (14) and (15), we obtain two equations for volume variable $U_{i, i}$ as

$$
\begin{aligned}
& {[2 G(1-v) /(1-2 v)] U_{i, i j j}=\rho_{s} U_{i, i t t},} \\
& \lim _{x_{j} \rightarrow \infty}\left(\tilde{U}_{i, i j} v_{j}-i k \tilde{U}_{i, i}\right)=0,
\end{aligned}
$$

which represents a pressure wave equation in a solid. Similarly, taking a curl operation on Eqs. (14) and (15), we obtain the other two equations for shear waves of variable $e_{r s i} U_{i, s}$, i.e.

$$
\begin{aligned}
& G e_{r s i} U_{i, s j j}=\rho_{s} e_{r s i} U_{i, s t t}, \\
& \lim _{x_{j} \rightarrow \infty}\left(e_{r s i} \tilde{U}_{i, s j} v_{j}-i k e_{r s i} \tilde{U}_{i, s}\right)=0 .
\end{aligned}
$$

The radiation condition defined above for solid dynamics is the same as the one for fluids. Therefore, we will only discuss the details for FS systems and directly extend the results to other cases.

\subsection{Fluid domain}

\section{Dynamic equation}

$\varphi_{, t t} / c^{2}=\varphi_{, i i}, \quad\left(x_{i}, t\right) \in \Omega_{f} \times\left(t_{1}, t_{2}\right)$.

Velocity-velocity potential relation

$v_{i}=\varphi_{, i}, \quad\left(x_{i}, t\right) \in \Omega_{f} \times\left(t_{1}, t_{2}\right)$.

Pressure-velocity potential relation

$p=-\rho_{f} \varphi_{, t}, \quad\left(x_{i}, t\right) \in \Omega_{f} \times\left(t_{1}, t_{2}\right)$.

\section{Boundary conditions}

Free surface:

$\varphi_{, i} \eta_{i}=-\varphi_{, t t} / g, \quad\left(x_{i}, t\right) \in \Gamma_{f} \times\left[t_{1}, t_{2}\right]$.

Fixed boundary:

$\varphi_{, i} \eta_{i}=0, \quad\left(x_{i}, t\right) \in \Gamma_{b} \times\left[t_{1}, t_{2}\right]$.

Radiation boundary:

$$
\lim _{r \rightarrow \infty} r^{\frac{h-1}{2}}\left(\phi_{, i} \eta_{i}-i k \phi\right)=0, \quad\left(x_{i}, t\right) \in \Gamma_{\infty} \times\left[t_{1}, t_{2}\right],
$$

or

$$
\lim _{r \rightarrow \infty} r^{\frac{h-1}{2}}\left(\varphi_{, i} \eta_{i}+\varphi_{, t} / \xi\right)=0,
$$

where $\varphi=\phi \mathrm{e}^{-i \omega t}$ and $r=\sqrt{x_{i} x_{i}}$ is assumed along the $\eta_{i}$ direction in the 3-dimensional case. The integrated speed $\xi$ of the wave is an unknown variable to be solved.

\subsection{Fluid-solid interaction interface}

$\varphi_{, i} \eta_{i}=-U_{i, t} v_{i}, \quad\left(x_{i}, t\right) \in \Sigma \times\left[t_{1}, t_{2}\right]$,

$\sigma_{i j} v_{j}=-p v_{i}, \quad\left(x_{i}, t\right) \in \Sigma \times\left[t_{1}, t_{2}\right]$.

\subsection{Displacement solution equations}

The displacement $U_{i}$ of the structure and the potential of velocity $\varphi$ of the fluid are chosen as variables to be determined, and the stress $\sigma_{i j}$ and strain $e_{i j}$ in the solid and the pressure $p$ of the fluid can be eliminated, so that we obtain the displacement-velocity potential equations of the system as follows.

Solid structure

$$
\begin{aligned}
& E_{i j k l} U_{k, l j}=\rho_{s} U_{i, t t}, \quad\left(x_{i}, t\right) \in \Omega_{s} \times\left(t_{1}, t_{2}\right), \\
& U_{i}=o, \quad\left(x_{i}, t\right) \in S_{u} \times\left[t_{1}, t_{2}\right], \\
& E_{i j k l} U_{k, l} \nu_{j}=0, \quad\left(x_{i}, t\right) \in S_{T} \times\left[t_{1}, t_{2}\right] .
\end{aligned}
$$

Fluid domain

$$
\begin{aligned}
& \varphi_{, t t} / c^{2}=\varphi_{, i i}, \quad\left(x_{i}, t\right) \in \Omega_{f} \times\left(t_{1}, t_{2}\right), \\
& \varphi_{, i} \eta_{i}=-\varphi_{, t t} / g, \quad\left(x_{i}, t\right) \in \Gamma_{f} \times\left[t_{1}, t_{2}\right], \\
& \varphi_{, i} \eta_{i}=0, \quad\left(x_{i}, t\right) \in \Gamma_{b} \times\left[t_{1}, t_{2}\right], \\
& \lim _{r \rightarrow \infty} r^{\frac{h-1}{2}}\left(\varphi_{, i} \eta_{i}+\varphi_{, t} / \xi\right)=0, \\
& \left(x_{i}, t\right) \in \Gamma_{\infty} \times\left[t_{1}, t_{2}\right] .
\end{aligned}
$$

Fluid-structure interaction interface

$$
\begin{aligned}
& \varphi_{, i} \eta_{i}=-U_{i, t} \nu_{i}, \quad\left(x_{i}, t\right) \in \Sigma \times\left[t_{1}, t_{2}\right], \\
& E_{i j k l} U_{k, l} \nu_{j}=\rho_{f} \varphi_{, t} \nu_{i}, \quad\left(x_{i}, t\right) \in \Sigma \times\left[t_{1}, t_{2}\right] .
\end{aligned}
$$

A trivial solution of $U_{i}=0=\varphi$ and $\omega=0$ satisfying Eqs. (27)-(35) represents a state of static equilibrium of the system, which is taken as the reference state of the motion of the system.

\section{A complex identity}

A complex power identity used in the following demonstrations is derived herein. Since the governing equations of the problem are a set of real coefficient equations, if a displacement $U_{i}$, potential of velocity $\varphi$ and natural frequency $\omega$ are a solution of Eqs. (27)-(35), the conjugate functions $U_{i}^{*}, \varphi^{*}$ 
and $\omega^{*}$ of this solution must also be a solution of the same equations [4]. The method of separation of variables is used to seek the solution of Eqs. (27)-(35) in the form

$U_{i}\left(x_{j}, t\right)=\tilde{U}_{i}\left(x_{j}\right) T(t), \quad \varphi\left(x_{j}, t\right)=\phi\left(x_{j}\right) T(t)$,

which are substituted into Eq. (30) giving

$T_{, t t}+\omega^{2} T=0, \quad \phi_{, i i}+\kappa^{2} \phi=0, \quad \kappa=\omega / c$,

where $\omega$ represents a natural frequency of the system to be determined. From Eq. (37) it follows that the possible solutions of function $T(t)$ take the form $\mathrm{e}^{-i \omega t}$ or $\mathrm{e}^{i \omega t}$. To satisfy the radiation condition (33) corresponding to condition (2), $T(t) \propto \mathrm{e}^{-i \omega t}$ is chosen. Therefore Eq. (36) takes the form

$U_{i}\left(x_{j}, t\right)=\tilde{U}_{i}\left(x_{j}\right) \mathrm{e}^{-i \omega t}, \quad \varphi\left(x_{j}, t\right)=\phi\left(x_{j}\right) \mathrm{e}^{-i \omega t}$.

The conjugate variables of Eq. (38)

$U_{i}^{*}\left(x_{j}, t\right)=\tilde{U}_{i}^{*}\left(x_{j}\right) \mathrm{e}^{i \omega^{*} t}, \quad \varphi^{*}\left(x_{j}, t\right)=\phi^{*}\left(x_{j}\right) \mathrm{e}^{i \omega^{*} t}$,

provide the solution of the conjugate equations, represented by stars "*", of Eqs. (27)-(35). We use displacement $U_{i}^{(I)}$, potential of velocity $\varphi^{(I)}$ and natural frequency $\omega^{(I)}$ representing a solution of Eqs. (27)-(35) with $U_{i}^{*(J)}, \varphi^{*(J)}$ and $\omega^{*(J)}$ denoting a conjugate solution of the conjugate equations, where superscripts $I$ and $J$ identify the number of natural modes of the system.

\subsection{A complex power identity}

Pre-multiplying Eq. (27) for the solution (I) by velocity $\dot{U}_{i}^{*}=i \omega^{*(J)} U_{i}^{*(J)}$, then integrating over volume $\Omega_{s}$ and using Green's theorem [21] and Eqs. (28), (29) and (34), (35), we obtain a complex power equation for the solid

$$
\begin{aligned}
& i \omega^{*(J)} \int_{\Sigma} \rho_{f} U_{i}^{*(J)} \varphi_{, t}^{(I)} v_{j} \mathrm{~d} S-i \omega^{*(J)} \int_{\Omega_{s}}\left(U_{i}^{*(J)} E_{i j k l} U_{k, l}^{(I)}\right. \\
& \left.+\rho_{S} U_{i}^{*(J)} U_{i, t t}^{(I)}\right) \mathrm{d} \Omega=0
\end{aligned}
$$

In a similar process, from Eqs. (30)-(33) and (34), (35) it follows that

$$
\begin{aligned}
& -i \omega^{*(J)}\left\{\int_{\Gamma_{f}} \rho_{f} \varphi^{*(J)}\left(\varphi_{, t t}^{(I)} / g\right) \mathrm{d} \Gamma\right. \\
& +\int_{\Gamma_{\infty}} \rho_{f} \varphi^{*(J)}\left(\varphi_{, t}^{(I)} / \xi\right) \mathrm{d} \Gamma+\int_{\Sigma} \rho_{f} \varphi^{*(J)} U_{i, t}^{(I)} v_{i} \mathrm{~d} \Gamma \\
& \left.+\int_{\Omega_{f}} \rho_{f}\left[\varphi_{, i}^{*(J)} \varphi_{, i}^{(I)}+\varphi^{*(J)} \varphi_{, t t}^{(I)} / c^{2}\right]\right\} \mathrm{d} \Omega=0 .
\end{aligned}
$$

For the conjugate equations (*) of Eqs. (27)-(35), we obtain

$$
\begin{aligned}
& -i \omega^{(I)} \int_{\Sigma} \rho_{f} U_{i}^{(I)} \varphi_{, t}^{*(J)} v_{j} \mathrm{~d} S+i \omega^{(I)} \int_{\Omega_{s}}\left(U_{i}^{(I)} E_{i j k l} U_{k, l}^{*(J)}\right. \\
& \left.+\rho_{s} U_{i}^{(I)} U_{i, t t}^{*(J)}\right) \mathrm{d} \Omega=0, \\
& i \omega^{(I)}\left\{\int_{\Gamma_{f}} \rho_{f} \varphi^{(I)}\left(\varphi_{, t t}^{*(J)} / g\right) \mathrm{d} \Gamma+\int_{\Gamma_{\infty}} \rho_{f} \varphi^{(I)}\left(\varphi_{, t}^{*(J)} / \xi\right) \mathrm{d} \Gamma\right. \\
& +\int_{\Sigma} \rho_{f} \varphi^{(I)} U_{i, t}^{*(J)} v_{i} \mathrm{~d} \Gamma+\int_{\Omega_{f}} \rho_{f}\left(\varphi_{, i}^{(I)} \varphi_{, i}^{*(J)}\right. \\
& \left.\left.+\varphi^{(I)} \varphi_{, t t}^{*(J)} / c^{2}\right) \mathrm{~d} \Omega\right\}=0 .
\end{aligned}
$$

From Eqs. (38) and (39), it follows that ()$_{, t}=-i \omega()$ and ()$_{, t}^{*}=i \omega^{*}()^{*}$ which when substituted into Eqs. (40)-(43) gives

$$
\begin{aligned}
& \omega^{(I)} \omega^{*(J)} \int_{\Sigma} \rho_{f} U_{i}^{*(J)} \varphi^{(I)} v_{i} \mathrm{~d} S \\
& -i \omega^{*(J)} \int_{\Omega_{s}}\left(U_{i, j}^{*(J)} E_{i j k l} U_{k, l}^{(I)}\right. \\
& \left.-\rho_{s} \omega^{2(I)} U_{i}^{*(J)} U_{i}^{(I)}\right) \mathrm{d} \Omega=0, \\
& i \omega^{*(J)} \omega^{2(I)}\left\{\int_{\Gamma_{f}}\left(\rho_{f} / g\right) \varphi^{*(J)} \varphi^{(I)} \mathrm{d} \Gamma\right. \\
& \left.+\int_{\Omega_{f}}\left(\rho_{f} / c^{2}\right) \varphi^{*(J)} \varphi^{(I)} \mathrm{d} \Omega\right\} \\
& -\omega^{*(J)} \omega^{(I)}\left\{\int_{\Gamma_{\infty}}\left(\rho_{f} / \xi\right) \varphi^{*(J)} \varphi^{(I)} \mathrm{d} \Gamma\right. \\
& \left.+\int_{\Sigma} \rho_{f} \varphi^{*(J)} U_{i}^{(I)} v_{i} \mathrm{~d} \Gamma\right\} \\
& =-i \omega^{*(J)} \int_{\Omega_{f}} \rho_{f} \varphi_{, i}^{*(J)} \varphi_{, i}^{(I)} \mathrm{d} \Omega=0, \\
& \omega^{(I)} \omega^{*(J)} \int_{\Sigma} \rho_{f} U_{i}^{(I)} \varphi^{*(J)} v_{i} \mathrm{~d} S \\
& +i \omega^{(I)} \int_{\Omega_{s}}\left(U_{i, j}^{(I)} E_{i j k l} U_{k, l}^{*(J)}\right. \\
& \left.-\omega^{2 *(J)} \rho_{s} U_{i}^{(I)} U_{i}^{*(J)}\right) \mathrm{d} \Omega=0,
\end{aligned}
$$




$$
\begin{aligned}
& -i \omega^{(I)} \omega^{2 *(J)}\left\{\int_{\Gamma_{f}}\left(\rho_{f} / g\right) \varphi^{*(J)} \varphi^{(I)} \mathrm{d} \Gamma\right. \\
& \left.+\int_{\Omega_{f}}\left(\rho_{f} / c^{2}\right) \varphi^{*(J)} \varphi^{(I)} \mathrm{d} \Gamma\right\} \\
& -\omega^{*(J)} \omega^{(I)}\left\{\int_{\Gamma_{\infty}}\left(\rho_{f} / \xi\right) \varphi^{*(J)} \varphi^{(I)} \mathrm{d} \Gamma\right. \\
& \left.+\int_{\Sigma} \rho_{f} \varphi^{(I)} U_{i}^{*(J)} v_{i} \mathrm{~d} \Gamma\right\} \\
& +i \omega^{(I)} \int_{\Omega_{f}} \rho_{f} \varphi_{, i}^{*(J)} \varphi_{, i}^{(I)} \mathrm{d} \Omega=0 .
\end{aligned}
$$

The summation of Eqs. (44)-(47) and application of the identity

$U_{i, j}^{*} E_{i j k l} U_{k, l}=U_{i, j} E_{i j k l} U_{k, l}^{*}$,

gives a complex power identity

$$
\begin{aligned}
& i\left(\omega^{(I)}-\omega^{*(J)}\right)\left\{\int _ { \Omega _ { s } } \left(U_{i, j}^{(I)} E_{i j k l} U_{k, l}^{*(J)}\right.\right. \\
& \left.+\rho_{s} \omega^{(I)} \omega^{*(J)} U_{i}^{(I)} U_{i}^{*(J)}\right) \mathrm{d} \Omega+\int_{\Omega_{f}}\left[\rho_{f} \varphi_{, i}^{(I)} \varphi_{, i}^{*(J)}\right. \\
& \left.+\left(\rho_{f} / c^{2}\right) \omega^{(I)} \omega^{*(J)} \varphi^{(I)} \varphi^{*(J)}\right] \mathrm{d} \Omega \\
& \left.+\int_{\Gamma_{f}}\left(\rho_{f} / g\right) \omega^{(I)} \omega^{*(J)} \varphi^{(I)} \varphi^{*(J)} \mathrm{d} \Gamma\right\} \\
& -2 \omega^{*(J)} \omega^{(I)} \int_{\Gamma_{\infty}}\left(\rho_{f} / \xi\right) \varphi^{*(J)} \varphi^{(I)} \mathrm{d} \Gamma=0 .
\end{aligned}
$$

\subsection{Physical explanation}

Equation (49) is a complex equation involving multiplications of complex variables. To understand it physically, we investigate the product of a complex sinusoidal force $f=\hat{F} \mathrm{e}^{-i \hat{\omega} t}$ of frequency $\hat{\omega}$ and its response velocity $v=$ $\hat{V} \mathrm{e}^{-i(\hat{\omega} t+\hat{\alpha})}$, where hats $\hat{\text { denote }}^{-}$real variables and $\hat{\alpha}$ a phase angle $\left(0 \leq \hat{\alpha} \leq 180^{\circ}\right)$ [12]. In the complex representation of a physical quantity, the real part represents the corresponding physical variable. For example the physical force $\hat{f}$ and velocity $\hat{v}$ are represented by the complex variables $f$ and $v$ as

$$
\begin{aligned}
& \hat{f}=\operatorname{Re} f=\hat{F} \cos \hat{\omega} t, \\
& \hat{v}=\operatorname{Re} \hat{v}=\hat{V} \cos (\hat{\omega} t+\hat{\alpha}) .
\end{aligned}
$$

The rate of work done by force $\hat{f}$ through velocity $\hat{v}$ is given by

$$
\begin{aligned}
& \hat{P}=\hat{f} \hat{v}=\hat{P}_{R}+\hat{P}_{I}, \\
& \hat{P}_{R}=\hat{F} \hat{V} \cos ^{2} \hat{\omega} \cos \hat{\alpha}, \\
& \hat{P}_{I}=-0.5 \hat{F} \hat{V} \sin 2 \hat{\omega} t \sin \hat{\alpha},
\end{aligned}
$$

of which the time averaged powers are [22]

$\overline{\hat{P}}=\overline{\hat{P}}_{R}+\overline{\hat{P}}_{I}, \quad \overline{\hat{P}}_{R}=0.5 \hat{F} \hat{V} \cos \hat{\alpha}, \quad \overline{\hat{P}}_{I}=0$.

The power of the force $\hat{f}$ consists of a real power $\hat{P}_{R}$ that has a non-zero time averaged power $\overline{\hat{P}}_{R}$ dissipated by the damping and a "workless power" $\hat{P}_{I}$ that describes the energy exchange in the system but has a zero time averaged power $\overline{\hat{P}}_{I}$.

The product of the complex force $f$ and the conjugate velocity $v^{*}$ is given by

$P=f v^{*}=\hat{F} \hat{V} \mathrm{e}^{i \hat{\alpha}}=2\left(\overline{\hat{P}}_{R}+i\left|\hat{P}_{I}\right|\right)$,

of which the real part equals two times the real time averaged power $\overline{\hat{P}}_{R}$ and the imaginary part equals two times the amplitude $\left|\hat{P}_{I}\right|$ of workless power $\hat{P}_{I}$. Therefore, physically, the real part of Eq. (49) gives an energy balance equation of time averaged power in the system and the imaginary part gives a relationship of the amplitudes of workless powers in the system.

\section{Characteristics of natural vibrations}

\subsection{Energy dissipative process}

Characteristic 1: Any non-trivial natural vibration of the FS System governed by Eqs. (7)-(26) undergoes an energy dissipative process although no damping exists in either solid or fluid materials.

To prove this, the complex power identity (49) is rewritten for a natural vibration $(I=J)$, therefore superscripts are omitted, in the following form

$$
\begin{aligned}
& \operatorname{Im}(\omega)\left\{\int_{\Omega_{s}}\left(U_{i, j} E_{i j k l} U_{k, l}^{*}+\rho_{s} \omega \omega^{*} U_{i} U_{i}^{*}\right) \mathrm{d} \Omega\right. \\
& +\int_{\Omega_{f}}\left[\rho_{f} \varphi_{, i} \varphi_{, i}^{*}+\left(\rho_{f} / c^{2}\right) \omega \omega^{*} \varphi \varphi^{*}\right] \mathrm{d} \Omega \\
& \left.+\int_{\Gamma_{f}}\left(\rho_{f} / g\right) \omega \omega^{*} \varphi \varphi^{*} \mathrm{~d} \Gamma\right\} \\
& +\omega^{*} \omega \int_{\Gamma_{\infty}}\left(\rho_{f} / \xi\right) \varphi^{*} \varphi \mathrm{d} \Gamma=0 .
\end{aligned}
$$


From the discussion in Sect. 4.2, the time averaged change rates of the mechanical energies of the system are:

Kinetic energy of solid

$$
\begin{aligned}
\overline{\dot{T}}_{s} & =0.5 \operatorname{Re}\left\{\int_{\Omega_{S}} \rho_{s} V_{i, t} V_{i}^{*} \mathrm{~d} \Omega\right\} \\
& =0.5 \int_{\Omega_{S}} \operatorname{Im}(\omega) \rho_{S} \omega \omega^{*} U U^{*} \mathrm{~d} \Omega,
\end{aligned}
$$

\section{Strain energy of solid}

$$
\begin{aligned}
\overline{\dot{\Pi}}_{s} & =0.5 \operatorname{Re}\left\{\int_{\Omega_{S}} U_{i, j t} E_{i j k l} U_{k, l}^{*} \mathrm{~d} \Omega\right\} \\
& =0.5 \int_{\Omega_{S}} \operatorname{Im}(\omega) U_{i, j t} E_{i j k l} U_{k, l}^{*} \mathrm{~d} \Omega,
\end{aligned}
$$

Kinetic energy of fluid

$$
\begin{aligned}
\overline{\dot{T}}_{f} & =0.5 \operatorname{Re}\left\{\int_{\Omega_{f}} \rho_{f} v_{i, t} v_{i}^{*} \mathrm{~d} \Gamma\right\} \\
& =0.5 \int_{\Omega_{f}} \operatorname{Im}(\omega) \rho_{f} \varphi_{, i} \varphi_{, i}^{*} \mathrm{~d} \Gamma \Omega,
\end{aligned}
$$

\section{Potential energy of fluid}

$$
\begin{aligned}
\bar{\Pi}_{f} & =0.5 /\left(\rho_{f} c^{2}\right) \operatorname{Re}\left\{\int_{\Omega_{f}} p_{, t} p^{*} \mathrm{~d} \Omega\right\} \\
& =\left(0.5 \rho_{f} / c^{2}\right) \operatorname{Re}\left\{\int_{\Omega_{f}} \varphi_{, t t} \varphi_{, t}^{*} \mathrm{~d} \Omega\right\} \\
& =\left(0.5 \rho_{f} / c^{2}\right) \int_{\Omega_{f}} \operatorname{Im}(\omega) \omega \omega^{*} \varphi \varphi^{*} \mathrm{~d} \Omega,
\end{aligned}
$$

Potential energy on free surface [23,24]

$$
\begin{aligned}
\overline{\dot{\Pi}}_{\Gamma} & =\left(0.5 \rho_{f} / g\right) \operatorname{Re}\left\{\int_{\Gamma_{f}} \varphi_{, t t} \varphi_{, t}^{*} \mathrm{~d} \Omega\right\} \\
& =\left(0.5 \rho_{f} / g\right) \int_{\Omega_{f}} \operatorname{Im}(\omega) \omega \omega^{*} \varphi \varphi^{*} \mathrm{~d} \Omega .
\end{aligned}
$$

Substituting Eqs. (55)-(59) into Eq. (54), we obtain

$$
\begin{aligned}
\overline{\dot{T}}_{S} & +\overline{\dot{\Pi}}_{S}+\overline{\dot{T}}_{f}+\overline{\dot{\Pi}}_{f}+\overline{\dot{\Pi}}_{\Gamma} \\
& =-0.5 \omega^{*} \omega \int_{\Gamma_{\infty}}\left(\rho_{f} / \xi\right) \varphi^{*} \varphi \mathrm{d} \Gamma \\
& =-0.5|\omega|^{2} \int_{\Gamma_{\infty}}\left(\rho_{f} / \xi\right)|\varphi|^{2} \mathrm{~d} \Gamma<0 .
\end{aligned}
$$

The left side of Eq. (60) represents the time averaged change rate of total mechanical energy of the system, which has a negative value since any non-trivial velocity potential $\varphi$ satisfying radiation condition (24) on an infinite boundary $\Gamma_{\infty}$ does not vanish. The energy of the system undergoes a dissipative process. For an undisturbed condition on the boundary $\Gamma_{\infty}$, no waves can reach the infinite boundary and therefore $\varphi=0$ on $\Gamma_{\infty}$. Equation (60) shows that the energy of the system is conservative [18].

This result reveals a physical fact that if a FS System undergoes a natural vibration excited by a disturbance which inputs an initial energy into the system, this energy will be dissipated by the radiation boundary $\Gamma_{\infty}$ of the fluid. In the solid, there is no energy dissipative mechanism and therefore its initial energy given by the disturbance must transfer into the fluid domain with a power $\int_{\Sigma}-p U_{i, t} \nu_{i} \mathrm{~d} S$ on the wet interface $\Sigma$. The energy flow balance equation of the fluid domain [22] concludes that the time averaged energy input from the solid into the fluid equals the time averaged energy dissipation on the radiation boundary, that is

$$
\begin{aligned}
\operatorname{Re} & \left\{\int_{\Sigma}-p U_{i, t}^{*} \nu_{i} \mathrm{~d} \Gamma\right\}=\operatorname{Re}\left\{\int_{\Gamma_{\infty}} \rho_{f} \varphi_{, t} U_{i, t}^{*} \nu_{i} \mathrm{~d} \Gamma\right\} \\
& =\left(\rho_{f} / \xi\right) \int_{\Gamma_{\infty}}|\omega|^{2}|\varphi|^{2} \mathrm{~d} \Gamma,
\end{aligned}
$$

where Eqs. (20), (24) and (25) are used to replace pressure $p$ by potential velocity $\varphi$.

\subsection{Complex conjugate eigenvalues}

Characteristic 2: All non-zero eigenvalues corresponding to natural frequencies of natural vibrations of the FS System governed by Eqs. (7)-(26) are complex conjugate numbers.

The proof of this conclusion is as follows. If the natural frequency $\omega$ is real, $\omega=\omega^{*}$, and from Eq. (54) it follows that

$$
\int_{\Gamma_{\infty}}\left(\rho_{f} / \xi\right) \varphi \varphi^{*} \mathrm{~d} \Gamma=\int_{\Gamma_{\infty}}\left(\rho_{f} / \xi\right)|\varphi|^{2} \mathrm{~d} \Gamma=0,
$$

which yields $|\varphi|=0$ on boundary $\Gamma_{\infty}$. However, $|\varphi|=$ 0 holds only for the trivial solution. Therefore, the natural frequency $\omega$ must be complex. Furthermore, Eqs. (7)-(26) 
or (27)-(35) are a set of equations with only real coefficients so that the conjugate frequency $\omega^{*}$ must be the corresponding natural frequency.

Characteristic 3: All non-zero complex natural frequencies $\omega$ in the solution form (38) of natural vibrations of the FS System governed by Eqs. (7)-(26) have negative imaginary parts.

It is easy to know that all terms in Eq. (54) are positive except $\operatorname{Im}(\omega)$. Therefore, the imaginary part $\operatorname{Im}(\omega)$ of the natural frequency $\omega$ must be negative to make Eq. (54) valid for a non-trivial solution of the problem. Naturally, $\operatorname{Im}\left(\omega^{*}\right)$ is positive.

\subsection{Number of conjugate eigenvalues}

Characteristic 4: The number of conjugate eigenvalues of the FS System defined by Eqs. (7)-(26) equals the number of degrees of freedom of the dry structure assuming no fluid in the system.

To confirm this, we investigate the displacement-velocity potential Eqs. (27)-(35) by separating them into the following two sub-problems. Sub-problem 1: a dynamic response problem of the solid structure governed by Eqs. (27)-(29) and excited by the traction force given in Eq. (35) on the fluid-structure interaction interface $\Sigma$ and Sub-problem 2: a radiation problem of the fluid domain governed by Eqs. (30)(33) and excited by the boundary velocity given in Eq. (34) on the fluid-structure interaction interface $\Sigma$.

For sub-problem 1, a Sturm-Liouville problem [4] is defined if the right side force of Eq. (35) vanishes, which represents the natural vibration of the dry solid structure. Assume that $\hat{\Omega}_{I}^{2}$ and $\bar{U}_{I i}(I=1,2,3, \ldots, N)$ are the first $N$ natural frequencies and the corresponding real natural modes of the dry solid structure. Based on the Sturm-Liouville theorem [4], these natural frequencies and modes satisfy the orthogonal relationships

$$
\begin{aligned}
& \int_{\Omega_{s}} \bar{U}_{J i, j} E_{i j k l} \bar{U}_{I k, l} \mathrm{~d} \Omega=\operatorname{diag}\left(\hat{\Omega}_{I}^{2}\right) \delta_{I J}, \\
& \int_{\Omega_{s}} \bar{U}_{J i} \rho_{S} \bar{U}_{I i} \mathrm{~d} \Omega=\delta_{I J},
\end{aligned}
$$

and all of the orthogonal natural modes construct a complete orthogonal function space. Therefore, any response $U_{i}$ of sub-problem 1 can be represented by a mode summation

$$
\begin{aligned}
U_{i} & =\overline{\boldsymbol{U}}_{i} \boldsymbol{q}(t), \\
\overline{\boldsymbol{U}}_{i} & =\left[\begin{array}{llll}
\bar{U}_{1 i} & \bar{U}_{2 i} & \cdots & \bar{U}_{N i}
\end{array}\right], \\
\boldsymbol{q}(t) & =\left[\begin{array}{llll}
q_{1} & q_{2} & \cdots & q_{N}
\end{array}\right]^{\mathrm{T}},
\end{aligned}
$$

where only the first $N$ modes are retained but not losing generality. Eq. (64) when substituted into Eq. (27) gives

$E_{i j k l} \overline{\boldsymbol{U}}_{k, l j} \boldsymbol{q}=\rho_{s} \overline{\boldsymbol{U}}_{i} \ddot{\boldsymbol{q}}$

Pre-multiplying Eq. (65) by $\overline{\boldsymbol{U}}_{i}^{\mathrm{T}}$ and then integrating over volume $\Omega_{s}$ using Green's theorem, orthogonal relationships (63) and boundary conditions (28), (29) and (35), we obtain

$\ddot{\boldsymbol{q}}+\operatorname{diag}\left(\hat{\Omega}_{I}^{2}\right) \boldsymbol{q}=\int_{\Sigma} \overline{\boldsymbol{U}}_{i}^{\mathrm{T}} \rho_{f} \varphi_{, t} \nu_{i} \mathrm{~d} S$.

Sub-problem 2 is a typical radiation problem of which the vibration source is the velocity on the boundary $\Sigma$ defined by Eq. (34). Based on the original Sommerfeld investigation $[1,2,4]$, a unique solution $\varphi$ of Sub-problem 2 exists. This solution satisfies the energy balance equation of the fluid given in Eq. (61), i.e.

$\operatorname{Re}\left\{\int_{\Sigma} \rho_{f} \varphi_{, t} U_{i, t}^{*} \nu_{i} \mathrm{~d} \Gamma\right\}=\left(\rho_{f} / \xi\right) \int_{\Gamma_{\infty}}|\omega|^{2}|\varphi|^{2} \mathrm{~d} \Gamma$.

Now, we seek the natural vibration of the FS System using Eqs. (66) and (67). As defined in Eq. (38), the time function vector $\boldsymbol{q}$ and the potential of velocity $\varphi$ take the forms

$\boldsymbol{q}=\boldsymbol{Q} \mathrm{e}^{-i \omega t}, \quad \varphi=\phi \mathrm{e}^{-i \omega t}$,

respectively, where $\boldsymbol{Q}=\left[\begin{array}{llll}Q_{1} & Q_{2} & \cdots & Q_{N}\end{array}\right]^{\mathrm{T}}$ represents a complex amplitude vector. Substituting Eq. (68) into Eqs. (66) and (67), we obtain

$$
\begin{aligned}
& \operatorname{diag}\left(\hat{\Omega}_{I}^{2}-\omega^{2}\right) \boldsymbol{Q}=-i \omega \rho_{f} \int_{\Sigma} \overline{\boldsymbol{U}}_{i}^{\mathrm{T}} v_{i} \phi \mathrm{d} S, \\
& \rho_{f}\left(\int_{\Sigma} \operatorname{Re}(\phi) v_{i} \overline{\boldsymbol{U}}_{i} \mathrm{~d} \Gamma\right) \boldsymbol{Q}=\left(\rho_{f} / \xi\right) \int_{\Gamma_{\infty}}|\varphi|^{2} \mathrm{~d} \Gamma .
\end{aligned}
$$

Equations (69), (70) can be rearranged in a matrix form as

$$
\left[\begin{array}{cc}
\operatorname{diag}\left(\hat{\Omega}_{I}^{2}-\omega^{2}\right) & i \omega \boldsymbol{R}^{\mathrm{T}} \\
\boldsymbol{R} & -d
\end{array}\right]\left[\begin{array}{l}
\boldsymbol{Q} \\
1
\end{array}\right]=0
$$

where

$$
\begin{aligned}
& \boldsymbol{R}=\rho_{f} \int_{\Sigma} \operatorname{Re}(\phi) \nu_{i} \overline{\boldsymbol{U}}_{i} \mathrm{~d} \Gamma, \\
& d=\left(\rho_{f} / \xi\right) \int_{\Gamma_{\infty}}|\varphi|^{2} \mathrm{~d} \Gamma>0 .
\end{aligned}
$$

Here, $\boldsymbol{R}$ is a coupling matrix of the FS System and $d$ involves the energy dissipative rate $|\omega|^{2} d(\mathrm{Nm} / \mathrm{s})$ on boundary $\Gamma_{\infty}$ of the system. The necessary and sufficient condition for 
Eq. (71) to have non-trivial solution $\boldsymbol{Q}$ requires its determinant of the coefficient matrix to vanish, which gives the characteristic equation of the FS System

$$
\left|\begin{array}{cc}
\operatorname{diag}\left(\hat{\Omega}_{I}^{2}-\omega^{2}\right) & i \omega \boldsymbol{R}^{\mathrm{T}} \\
\boldsymbol{R} & -d
\end{array}\right|=0
$$

Equation (73) is a complex algebraic equation of the unknown variable $\omega$. The highest power of $\omega$ is $2 N$ which defines the number of pairs of complex conjugate solutions $\omega$ equals $N$, which is the degree of freedom of the dry solid structure in the system.

\subsection{Orthogonal relationships}

Based on the identity (49), we investigate the orthogonal relationships of natural modes of the system.

\section{Undisturbed boundary condition on $\Gamma_{\infty}$}

For this case, the potential velocity $\varphi$ on $\Gamma_{\infty}$ vanishes and therefore the integration defined on $\Gamma_{\infty}$ in Eq. (49) vanishes. For any two different natural frequencies $\omega^{(I)}$ and $\omega^{(J) *}$, it follows that

$$
\begin{aligned}
& \int_{\Omega_{s}} U_{i, j}^{(I)} E_{i j k l} U_{k, l}^{*(J)} \mathrm{d} \Omega+\int_{\Omega_{f}} \rho_{f} \varphi_{, i}^{(I)} \varphi_{, i}^{*(J)} \mathrm{d} \Omega=0, \\
& \int_{\Omega_{s}} \rho_{s} U_{i}^{*(J)} U_{i}^{(I)} \mathrm{d} \Omega+\int_{\Gamma_{f}}\left(\rho_{f} / g\right) \varphi^{*(J)} \varphi^{(I)} \mathrm{d} \Gamma \\
& \quad+\left(\rho_{f} / c^{2}\right) \int_{\Omega_{f}} \varphi^{*(J)} \varphi^{(I)} \mathrm{d} \Omega=0,
\end{aligned}
$$

which are two orthogonal relationships of the fluid-structure interaction system with an undisturbed condition on the infinite boundary $\Gamma_{\infty}$. Xing et al. [18] demonstrated this orthogonal relationship for beam-water interaction systems. As discussed in Sect. 4.2, Eqs. (74) and (75) are two relationships of the workless powers in the system. Physically, the time averaged power done by the inertial and elastic forces of a natural vibration of the system on the motion of another natural vibration of the system vanishes.

\section{Radiation case}

In this case, Eq. (49) is a complex equation including two real equations. The real part represents an equilibrium equation of the time averaged power dissipated by the forces of mode $(J)$ through the motion of mode $(I)$ (or in a reverse direction) but the imaginary part of the equation represents a relationship of the amplitudes of workless powers of the system. For radiation cases, there is no simple real orthogonal equation being found.

\section{$6 \mathrm{SS}$ and FSS systems}

\subsection{SS system}

As defined in Sect. 2, an essential difference between an SS System and an FS System is the location of the Sommerfeld condition. For an SS System, the Sommerfeld condition is located only on the solid boundary. The SS System is a reverse case of the FS System. It is not difficult to derive similar theorems and conclusions as described for FS Systems. We neglected similar results for this system. For example the characteristic 4 in Sect. 5.3 now reads that the number of conjugate natural frequencies of the SS System equals the number of degrees of freedom of the fluid domain with assumed rigid wet boundaries in the system.

\subsection{FSS system}

For FSS systems, there exist the Sommerfeld boundary conditions on both the solid and fluid boundaries. We consider two radiation problems, one in a fluid and another in a solid, excited by a disturbance on interface $\Sigma$. Based on Sommerfeld's original investigation [1,2], a unique solution $\varphi$ in the fluid and $U_{i}$ in the solid exists. Therefore, if the disturbance on interface $\Sigma$ vanishes, this gives a unique trivial natural vibration, which implies that there are no non-trivial natural vibrations existing for the FSS System.

\section{Examples}

To help readers understand the generalized conclusions theoretically demonstrated above, it is useful to summarise the two examples by Xing [20] in Sects. 7.1 and 7.2. The interested reader may wish to consult this reference for more details of the solution process.

\subsection{A mass-spring system coupled to an infinite fluid} domain

Figure 2 shows a spring-mass system of stiffness $K$ and mass $M$ is coupled to a 1-D straight semi-infinite long fluid channel of section area $S$. The fluid of mass density $\rho$ is assumed compressible and non-viscous. The origin of the $x$ axis is located at the natural equilibrium position of the mass. The displacement $\tilde{x}(t)$ of the mass and the pressure $p(x, t)$ of the fluid are used as variables.

The characteristic equation of the system was obtained as

$\omega^{2}+2 \eta i \omega-\hat{\Omega}^{2}=0$,

where $\hat{\Omega}=\sqrt{K / M}$ represents the natural frequency of the mass-spring system and $\eta=0.5 \mathrm{~S} \rho \mathrm{c} / \mathrm{M}$. The solutions of 


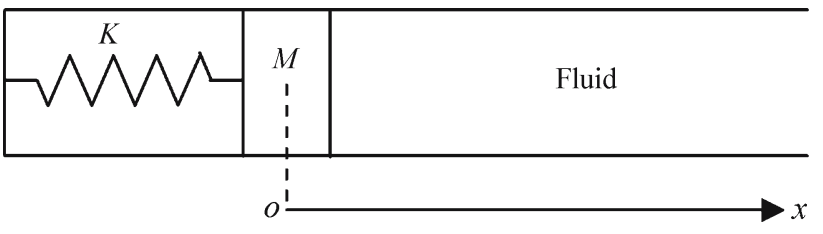

Fig. 2 A one dimensional fluid-mass-spring interaction system

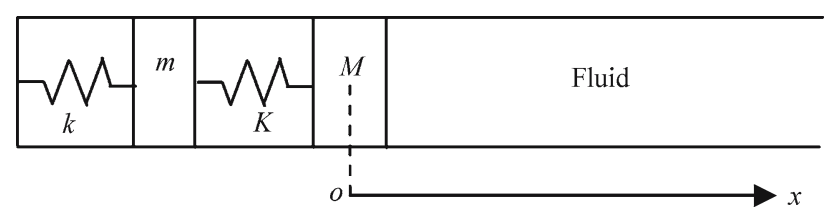

Fig. 3 A mass-spring system of two degrees of freedom coupled to an infinite fluid domain

Eq. (76) are

$\omega=-i \eta \pm \Omega, \quad \Omega=\sqrt{\hat{\Omega}^{2}-\eta^{2}}$,

being the natural frequency of the system which corresponds to a pair of conjugate eigenvalues $i \omega=\eta \pm i \Omega$. The corresponding complex natural mode of the system was obtained as

$$
\begin{aligned}
& p(x, t)=\rho c(-\eta-i \Omega) \mathrm{e}^{\frac{\eta}{c}(x-c t)} \mathrm{e}^{+i \frac{\Omega}{c}(x-c t)}, \\
& \tilde{x}(t)=\mathrm{e}^{-\eta t} \mathrm{e}^{-i \Omega t} .
\end{aligned}
$$

The real pressure $\hat{p}$ and displacement $\hat{x}$ of the system are

$$
\begin{aligned}
\hat{p}= & \rho c \mathrm{e}^{\frac{\eta}{c}(x-c t)}\{-\eta \cos [\Omega(x-c t) / c] \\
& +\Omega \sin [\Omega(x-c t) / c]\}, \\
\hat{x}= & \mathrm{e}^{-\eta t} \cos \Omega t .
\end{aligned}
$$

This solution confirms the general conclusions described in Sect. 5.

\subsection{A system of two degrees of freedom coupled to an} infinite fluid domain

Figure 3 shows another simple system extended from Fig. 2. The solution of the natural vibration is sought in the form

$$
\begin{aligned}
& y(t)=Y \mathrm{e}^{-i \omega t}, \quad \tilde{x}(t)=X \mathrm{e}^{-i \omega t}, \\
& p(x, t)=P(x) \mathrm{e}^{-i \omega t},
\end{aligned}
$$

To reveal the dynamic behaviour of the system, a mode transformation

$$
\left[\begin{array}{l}
y \\
\tilde{x}
\end{array}\right]=\boldsymbol{\Phi} \boldsymbol{q}=\left[\begin{array}{ll}
\varphi_{11} & \varphi_{12} \\
\varphi_{21} & \varphi_{22}
\end{array}\right]\left[\begin{array}{l}
q_{1} \\
q_{2}
\end{array}\right]
$$

is introduced. Here $\boldsymbol{\Phi}$ and $\boldsymbol{q}$ are the matrix of normalized mode vectors and the generalized coordinator vector of the dry solid system, respectively. The matrix $\boldsymbol{\Phi}$ satisfies the normalization relationships

$\boldsymbol{\Phi}^{\mathrm{T}} \boldsymbol{M} \boldsymbol{\Phi}=\boldsymbol{I}, \quad \boldsymbol{\Phi}^{\mathrm{T}} \boldsymbol{K} \boldsymbol{\Phi}=\boldsymbol{\Lambda}$,

where $\boldsymbol{I}$ denotes a unit matrix and $\boldsymbol{\Lambda}=\operatorname{diag}\left(\hat{\Omega}_{1}^{2}, \hat{\Omega}_{2}^{2}\right)$ representing a diagonal matrix of the square of two natural frequencies of the dry structure. The dynamic equation describing the natural vibration of this system was transformed into the following mode form

$\boldsymbol{I} \ddot{\boldsymbol{q}}+\boldsymbol{\Lambda} \boldsymbol{q}=-\operatorname{Sp}(0, t)\left[\begin{array}{l}\varphi_{21} \\ \varphi_{22}\end{array}\right]$.

The mode transformation does not change the eigenvalues of the system [4], so that the natural frequencies of the system can be obtained by solving Eq. (83). Now the solution (80) takes the form

$q_{1}(t)=Q_{1} \mathrm{e}^{-i \omega t}, \quad q_{2}(t)=Q_{2} \mathrm{e}^{-i \omega t}, \quad p(x, t)=P(x) \mathrm{e}^{-i \omega t}$

In the end, the equation describing the natural vibration of the system is represented as

$\left[\begin{array}{ccc}\hat{\Omega}_{1}^{2}-\omega^{2} & 0 & S \varphi_{21} \\ 0 & \hat{\Omega}_{2}^{2}-\omega^{2} & S \varphi_{22} \\ \rho c \omega^{2} \varphi_{21} & \rho c \omega^{2} \varphi_{22} & -i \omega\end{array}\right]\left[\begin{array}{c}Q_{1} \\ Q_{2} \\ a\end{array}\right]=0$,

of which the determinant of the coefficient matrix vanishes giving the characteristic equation of this system

$$
\begin{gathered}
\left(1-\lambda^{2}\right)\left(1-\lambda^{2} / \alpha^{2}\right)-2 i \varepsilon_{1} \lambda\left(1-\lambda^{2} / \alpha^{2}\right) \\
-2 i \varepsilon_{2}(\lambda / \alpha)\left(1-\lambda^{2}\right)=0,
\end{gathered}
$$

where

$$
\begin{array}{ll}
\lambda=\omega / \hat{\Omega}_{1}, & \alpha=\hat{\Omega}_{2} / \hat{\Omega}_{1}, \\
\varepsilon_{1}=0.5 \operatorname{S\rho } \rho \varphi_{21}^{2} / \hat{\Omega}_{1}, & \varepsilon_{2}=0.5 \operatorname{S\rho c} \varphi_{22}^{2} / \hat{\Omega}_{2} .
\end{array}
$$

It was demonstrated that: (i) the solutions of Eq. (86) are complex only and they consist of two pairs of conjugate complex numbers, (ii) the number of natural modes of the system equals the number of degrees of freedom of the dry solid system and it is independent of the infinite fluid domain, (iii) the natural frequency $\omega$ of the system must have a negative imaginary part.

\subsection{An FSS system}

Figure 4 shows a one dimensional FSS System consisting of a semi-infinite uniform elastic rod coupled to a semi-infinite fluid domain, the same as in Fig. 3. The mass density and elastic modulus of the rod are denoted by $\rho_{S}$ and $E$, respectively. Here, the rod has an infinite number of dry natural modes. The governing equations of the system are as follows. Solid domain

$$
\begin{aligned}
& \partial^{2} u / \partial x^{2}=\left(1 / C^{2}\right) \partial^{2} u / \partial t^{2}, \\
& C=\sqrt{E / \rho_{s}}, \quad-\infty<x<0 .
\end{aligned}
$$




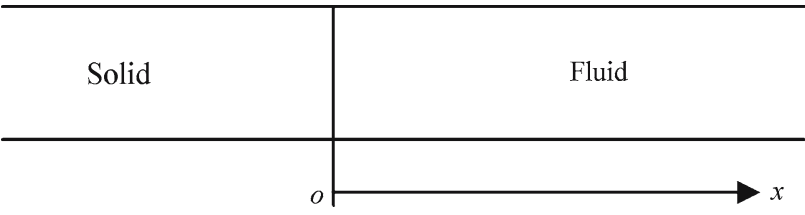

Fig. 4 An FSS System consisting of an infinite elastic rod coupled to an infinite long fluid domain

\section{Interaction conditions}

$E S(\partial u / \partial x)(0, t)=-p(0, t)$,

$\partial p / \partial x=-\rho_{f} \partial^{2} u / \partial t^{2}, \quad x=0$.

Fluid domain

$\frac{\partial^{2} p}{\partial x^{2}}=\frac{1}{c^{2}} \frac{\partial^{2} p}{\partial t^{2}}, \quad(0<x<\infty)$.

Sommerfeld conditions

$\frac{\partial p}{\partial x}=(i \omega / c) P, \quad x \rightarrow \infty$,

$\partial U / \partial x=-(i \omega / C) U, \quad x \rightarrow-\infty$.

We seek the natural vibrations $(\omega \neq 0)$ in the forms

$u(x, t)=U(x) \mathrm{e}^{-i \omega t}, \quad p(x, t)=P(x) \mathrm{e}^{-i \omega t}$,

satisfying Eqs. (88)-(93). These solutions take the form

$u(x, t)=A \mathrm{e}^{-i \omega x / c} \mathrm{e}^{-i \omega t}, \quad p(x, t)=B \mathrm{e}^{i \omega x / c} \mathrm{e}^{-i \omega t}$,

where $A$ and $B$ are two constants. Substituting Eq. (95) into Eqs. (89) and (90), we obtain

$\left[\begin{array}{cc}i & -\rho_{f} c \omega \\ 1 & -i E S \omega / C\end{array}\right]\left[\begin{array}{l}p \\ u\end{array}\right]=0$

of which the necessary and sufficient condition of existing non-zero solutions requires

$E S \omega / C+\rho_{f} c \omega=0$,

which is impossible. Therefore, there are no natural vibrations for this FSS System, which support the conclusion described previously.

\subsection{A 2-dimensional example}

Let us consider a fluid-solid interaction problem as shown in Fig. 5. The system consists of a mass-spring system and a two dimensional water domain $\Omega_{f}$ of depth $H$ and thickness $B$ perpendicular to the paper plane. The fluid domain boundaries include a free surface $\Gamma_{f}$, infinite boundary $\Gamma_{\infty}$ and bottom $\Gamma_{b}$. We assume that the mass $M$ moves only in the $x$ direction and choose the static equilibrium position $O$ of the mass as the origin of reference coordinate system $O-x y$.

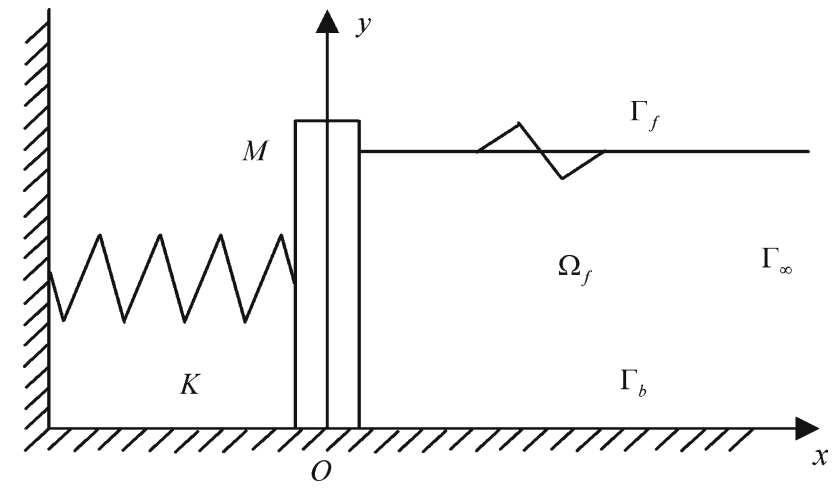

Fig. 5 A 2-dimensional example involving both free surface and pressure waves

Using the displacement $U$ of the mass and the pressure $p$ of the water relative to its static equilibrium configuration, we seek a natural vibration of the system in the form

$$
\begin{aligned}
& U=\tilde{U} \mathrm{e}^{-i \omega t}, \\
& p=P(x, y) \mathrm{e}^{-i \omega t}=X(x) Y(y) \mathrm{e}^{-i \omega t},
\end{aligned}
$$

where $\omega$ denotes a complex natural frequency of the system. This solution satisfies the governing equations of the system.

$$
\partial^{2} p / \partial x^{2}+\partial^{2} p / \partial y^{2}=\left(1 / c^{2}\right) \partial^{2} p / \partial t^{2}, \quad(x, y) \in \Omega_{f},
$$

$\partial p / \partial y=(-1 / g) \partial^{2} p / \partial t^{2}, \quad y=H$,

$\partial p / \partial y=0, \quad y=0$

$\partial P / \partial x-i k P=0, \quad x \rightarrow \infty$,

$M \ddot{U}+K U=-B \int_{0}^{H} p(0, y, t) \mathrm{d} y$,

$\partial p / \partial x=-\rho \ddot{U}, \quad x=0$.

Equation (102) is a Sommerfeld condition and $k$ denotes a characteristic parameter of the system to be determined later. Substituting Eq. (98) into Eqs. (100)-(103), we obtain

$Y^{\prime \prime}+\lambda^{2} Y=0$

$Y^{\prime}(0)=0$,

$Y^{\prime}(H)-\left(\omega^{2} / g\right) Y(H)=0$.

$X^{\prime \prime}+k^{2} X=0$,

$X^{\prime}-j k X=0, \quad x \rightarrow \infty$.

$\omega^{2} / c^{2}=k^{2}+\lambda^{2}$.

The function $Y$ satisfying Eq. (105) takes the form [18]

$Y_{n}(y)=\cos \left(\lambda_{n} y\right), \quad n=1,2,3, \ldots$,

where $\lambda_{n}$ are the solutions of equation

$\lambda_{n} \tan \left(\lambda_{n} H\right)=-\omega^{2} / g$. 
The functions $Y_{n}(y)$ satisfy the following orthogonal relationship

$$
\int_{0}^{H} Y_{m} Y_{n} \mathrm{~d} y=\left\{\begin{array}{cc}
0, & m \neq n, \\
{\left[2 \lambda_{n} H+\sin \left(2 \lambda_{n} H\right)\right] /(4 \lambda),} & m=n,
\end{array}\right.
$$

and the corresponding parameters $k_{n}$ are required to satisfy Eq. (107), i.e.

$\omega^{2} / c^{2}=k_{n}^{2}+\lambda_{n}^{2}, \quad n=1,2,3, \ldots$

The function $X_{n}$ satisfying Eq. (106) takes the form

$$
X_{n}(x)=\mathrm{e}^{i k_{n} x} .
$$

The pressure in the fluid is now expressed as a summation in the form

$p=P(x, y) \mathrm{e}^{-i \omega t}=\sum_{n=1,2, \ldots} P_{n} \mathrm{e}^{i k_{n} x} \cos \left(\lambda_{n} y\right) \mathrm{e}^{-i \omega t}$,

where $P_{n}$ are constants to be determined. Now a natural frequency $\omega \neq 0$ is sought. From Eqs. (103) and (104), it follows that

$$
\left(\hat{\Omega}^{2}-\omega^{2}\right) \tilde{U}+(B / M) \sum_{n=1,2,3, \ldots} P_{n} \int_{0}^{H} \cos \left(\lambda_{n} y\right) \mathrm{d} y=0,
$$

$\hat{\Omega}^{2}=K / M$,

$\omega^{2} \rho \tilde{U}-\sum_{n=1,2,3, \ldots} i k_{n} P_{n} \cos \left(\lambda_{n} y\right)=0$.

Using the orthogonal relationship (110) to Eq. (115), we derive

$$
\begin{aligned}
& \omega^{2} \rho \tilde{U} \int_{0}^{H} \cos \left(\lambda_{n} y\right) \mathrm{d} y \\
& -i k_{n} P_{n} \int_{0}^{H} \cos ^{2}\left(\lambda_{n} y\right) \mathrm{d} y=0, \quad n=1,2,3, \ldots,
\end{aligned}
$$

Equations (114) and (116) are combined to a set of algebraic equations to seek non-trivial solutions of $\tilde{U}$ and $P_{n}(n=$ $1,2,3, \ldots)$. Retaining the first $N$ functions $Y_{n}(x)$, we express this set of equations in the matrix form

$$
\left[\begin{array}{cccc}
\left(\omega^{2}-\hat{\Omega}^{2}\right) M & -B \bar{Y}_{1} & \cdots & -B \bar{Y}_{N} \\
\omega^{2} \rho \bar{Y}_{1} & -i k_{1} \hat{Y}_{1} & \cdots & 0 \\
\vdots & \vdots & \ddots & \vdots \\
\omega^{2} \rho \bar{Y}_{N} & 0 & \cdots & -i k_{N} \hat{Y}_{N}
\end{array}\right]\left[\begin{array}{c}
\tilde{U} \\
P_{1} \\
\vdots \\
P_{N}
\end{array}\right]=0
$$

where

$$
\begin{aligned}
\bar{Y}_{n} & =\int_{0}^{H} \cos \left(\lambda_{n} y\right) \mathrm{d} y=\sin \left(\lambda_{n} H\right) / \lambda_{n}, \\
\hat{Y}_{n} & =\int_{0}^{H} \cos ^{2}\left(\lambda_{n} y\right) \mathrm{d} y=\left[2 \lambda_{n} H+\sin \left(2 \lambda_{n} H\right)\right] /\left(4 \lambda_{n}\right), \\
n & =1,2,3, \ldots
\end{aligned}
$$

The necessary and sufficient condition for Eq. (117) to have a non-trivial solution requires that the determinant of the coefficient matrix of Eq. (117) vanishes, i.e.

$$
\left|\begin{array}{cccc}
\left(\hat{\Omega}^{2} / \omega^{2}-1\right) M / \rho & B \bar{Y}_{1} & \cdots & B \bar{Y}_{N} \\
\bar{Y}_{1} & -j k_{1} \hat{Y}_{1} & \cdots & 0 \\
\vdots & \vdots & \ddots & \vdots \\
\bar{Y}_{N} & 0 & \cdots & -j k_{N} \hat{Y}_{N}
\end{array}\right|=0 .
$$

Equation (119) is the characteristic equation of the system from which the natural frequency of the system can be determined. To solve this complex equation in association with Eqs. (109) and (111), a numerical iteration method is required. For a starting value of $\tilde{\omega}, \lambda_{n}$ and the corresponding $k_{n}$ are obtained from Eqs. (109) and (111), which are then substituted into Eq. (119) to obtain a new value of $\omega$. Interactions continue until an accepted error criterion $|\omega-\tilde{\omega}| \leq \varepsilon$ is reached. For each set of $\lambda_{n}$ and $k_{n}$, Eq. (119) is an algebraic equation of $\omega$. The highest power of $\omega$ is 2 and, therefore, a complex conjugate $\omega$ can be found.

To avoid complex numerical calculations, we consider a special case assuming that the depth $H$ of water is very small, i.e. shallow water. In this case, Eqs. (119) and (118) give a solution

$\lambda^{2}=-\omega^{2} /(g H), \quad \bar{Y}=H=\hat{Y}$,

due to $\tan \left(\lambda_{n} H\right) \approx \lambda_{n} H \approx \sin \left(\lambda_{n} H\right)$. This result when substituted into Eq. (111) yields

$$
\begin{aligned}
& {\left[\left(c^{2}+g H\right) \omega^{2}\right] /\left(c^{2} g H\right)=\omega^{2} / \xi^{2}=k^{2},} \\
& \xi=\sqrt{(g H) /\left(1+g H / c^{2}\right)} .
\end{aligned}
$$

The corresponding Eq. (119) now takes the form

$$
\left|\begin{array}{cc}
\left(\hat{\Omega}^{2} / \omega^{2}-1\right) M / \rho & B H \\
H & -i k H
\end{array}\right|=0 .
$$

The characteristic equation of the system is

$$
\begin{aligned}
& \bar{\omega}^{2}+2 i \bar{\omega} \eta-1=0, \\
& \bar{\omega}=\omega / \hat{\Omega}, \quad \eta=\rho B H \xi /(2 M \hat{\Omega}),
\end{aligned}
$$

which has the solution

$$
\bar{\omega}=-i \eta \pm \sqrt{1-\eta^{2}},
$$


and the corresponding mode form ( $\tilde{U}=1$ for normalization)

$\tilde{U}=1, \quad P=-i \rho \bar{\omega} \hat{\Omega} \xi$.

Here, the parameter $\eta$ plays the role of damping factor and the parameter $\xi$ represents the speed of the radiation wave influenced by the free surface and pressure waves. A further discussion on it is as follows:

(i) Nofree surface waves. If free surface waves are not considered, the gravity acceleration tends to infinity, so that the speed of radiation wave $\xi \rightarrow c$, and $k=\kappa=\omega / c$, which is the case considering only the pressure wave. Increasing speed of sound $c$ implies that the energy dissipates faster from the radiation boundary, so that damping factor $\eta$ is larger.

(ii) Incompressible water. Assume that the water is incompressible. The speed $c$ of sound tends to infinity. Therefore, $\xi \rightarrow \sqrt{g H}$ and $k=\omega / \sqrt{g H}$ where only the free surface wave is considered.

This example addresses the fail reason by Zhao [19]. If both of the free surface wave and the compressible wave are considered, the speed of radiation wave does not equal the speed of sound in water. Here we introduce a generalized parameter representing the speed of radiation wave in the Sommerfeld condition (2). While preparing this paper, following the finding in this example, a separate paper by Xing [25] was completed to investigate the natural vibration of beam-water interaction systems subject to the Sommerfeld condition given in Eq. (2).

\section{Dynamic responses}

The complex mode theory developed in matrix theory in mathematics can be used to solve the dynamic responses of a dynamic system. For example Zhou and Wang [26] used this approach to solve the dynamic response of offshore platforms subject to the hydrodynamic forces represented by the Morison's approximate equation [7] which introduces the water added mass and damping into the dry structure equations. Cui et al. [27] solved the dynamic response of a parallel-plate fuel assembly excited by the fluid flow of a constant speed. The added mass and damping produced by the flow was added in the structure equations, so that a complex dynamic equation of the system was produced and solved by a numerical method.

The above examples confirm that the dynamic response of a Sommerfeld system can be solved using the complex mode theory in mathematics. However, as discussed in Sect. 5, since there is not a simple orthogonal relationship of the natural modes of a Sommerfeld system, it is not convenient to use the complex modes in a mode summation method for dynamic response analysis. As is well known, the natural modes of the dry structure construct a complete orthogonal function space to describe any motion of the solid. These natural modes of the dry structure are easily obtained by a finite element analysis [13,14]. Furthermore, as indicated by Characteristic 4 of the FS Sommerfeld system, the number of its complex modes equals the degrees of freedom of the involved dry structure. Therefore, the space of the natural modes of the dry structure and the corresponding fluid pressure forms are sufficient to describe any dynamic responses of FS Systems. This conclusion theoretically confirms that the solution approach to solve free surface water wave radiation problems using the dry modes of the ship [7] is sufficient. To explain this, we consider the example shown in Fig. 3. Assume that a force $F \mathrm{e}^{-i \hat{\Omega} t}$ is applied on the mass $m$, so that the dynamic equation of this system is represented as

$$
\left[\begin{array}{cc}
m & 0 \\
0 & M
\end{array}\right]\left[\begin{array}{c}
\ddot{y} \\
\ddot{\tilde{x}}
\end{array}\right]+\left[\begin{array}{cc}
k+K & -K \\
-K & K
\end{array}\right]\left[\begin{array}{c}
y \\
\tilde{x}
\end{array}\right]=\left[\begin{array}{c}
F \mathrm{e}^{-i \hat{\Omega} t} \\
-S p(0, t)
\end{array}\right] .
$$

The mode transformation given in Eq. (81) transforms Eq. (126) into the mode form

$$
\boldsymbol{I} \ddot{\boldsymbol{q}}+\boldsymbol{\Lambda} \boldsymbol{q}=\left[\begin{array}{ll}
\varphi_{11} & \varphi_{21} \\
\varphi_{12} & \varphi_{22}
\end{array}\right]\left[\begin{array}{c}
F \mathrm{e}^{-i \hat{\Omega} t} \\
-S p(0, t)
\end{array}\right] .
$$

Since the system is linear, the frequency of dynamic responses is same as the frequency of the external force. The forced vibration of the system is assumed as

$q(t)=Q \mathrm{e}^{-i \hat{\Omega} t}, \quad p(x, t)=a \mathrm{e}^{i \hat{\Omega} x / c} \mathrm{e}^{-i \hat{\Omega} t}$,

which when substituted into Eq. (127) produces

$$
\left[\begin{array}{ccc}
\hat{\Omega}_{1}^{2}-\hat{\Omega}^{2} & 0 & S \varphi_{21} \\
0 & \hat{\Omega}_{2}^{2}-\hat{\Omega}^{2} & S \varphi_{22} \\
\rho c \hat{\Omega} \varphi_{21} & \rho c \hat{\Omega} \varphi_{22} & -i
\end{array}\right]\left[\begin{array}{c}
Q_{1} \\
Q_{2} \\
a
\end{array}\right]=\left[\begin{array}{c}
\varphi_{11} \\
\varphi_{12} \\
0
\end{array}\right] F
$$

The force frequency $\hat{\Omega}$ is a real number which cannot equal any complex natural frequencies $\omega$ of the Sommerfeld system. As result of this, the determinant of the coefficient matrix of Eq. (129) does not vanish and the solution $Q_{1}, Q_{2}$ and $a$ can be uniquely determined. The physical dynamic response of the system can be obtained from Eq. (81). This example shows that for the dynamic response analysis of FS Systems, it is sufficient to represent the solid motion of the system based on the natural modes of the dry structure. The complex natural mode analysis of the total system may not be necessary. 


\section{Conclusions}

The theoretical demonstrations and the selected examples in this paper confirm and reveal the following generalized conclusions:

(1) The natural vibration of a generalized FS system behaves as free damped vibrations although there is no material damping in both the solid and the fluid. The damping is caused by the Sommerfeld radiation condition at the infinite boundary where the energy of the system transmits from inside to outside.

(2) The natural vibration of a generalized FS system is governed by a complex eigenvalue problem having only complex conjugate eigenvalues. The number of complex conjugate eigenvalues of the system equals the number of degrees of freedom of the solid structure in the system but independent of the fluid domain where the Sommerfeld condition is imposed.

(3) There exists a complex energy flow identity for any two different natural vibrations. The real part of this identity represents an equilibrium equation of the time averaged power dissipated through the natural vibration motions. The imaginary part represents a workless power relationship between the two natural vibrations.

(4) The natural modes, satisfying its orthogonal relationships, of the dry structure of an FS system are sufficient to be used as a set of complete and orthogonal functions to construct a mode space to represent the dynamic response of the FS system excited by external forces. This is an efficient approach for dynamic response analysis of Sommerfeld systems by avoiding solving any complex eigenvalue problems.

(5) For SS Systems, the reverse conclusions can be made. For FSS Systems, there exist no natural vibrations.

(6) The new parameter representing the speed of radiation wave in the Sommerfeld condition is introduced, which provides an approach to investigate some complicated radiation problems involving various boundary conditions. As a result of this, the natural vibrations of Sommerfeld systems involving both free surface and compressible waves can be solved.

\section{References}

1. Sommerfeld, A.: Die Greensche Funktionen der Schwingungsgleichung. Jahresber Deutsch Math. Verein 21, 309-353 (1912)

2. Somerfield, A.: Partial Differential Equations in Physics. Academic, New York (1949)

3. Rellich, F.: Über das asymptotische Verhalten der Losungen von $\Delta u+\lambda u=0$ in unendlichen Gebieten. Jahresber Deutsch Math. Verein 53, 57-65 (1943)
4. Courant, R., Hilbert, D.: Methods of Mathematical Physics. Interscience, New York (1962)

5. Magnus, W., Oberhettinger, F.: Formulas and Theorems for the Special Functions of Mathematical Physics. Chelsea Publishing Co, New York (1949)

6. Newman, J.N.: The theory of ship motions. Adv. Appl. Mech. 18, 221-283 (1978)

7. Bishop, R.E.D., Price, W.G.: Hydroelasticity of Ships. Cambridge University Press, London (1979)

8. Eatock Taylor, R.: A review of hydrodynamic load analysis for submerged structures excited by earthquakes. Eng. Struct. 3, 131139 (1981)

9. Gaunaurd, G.C., Brill, D.: Acoustic spectrogram and complexfrequency poles of a resonantly excited elastic tube. J. Acoust. Soc. Am. 75, 1680-1693 (1984)

10. Tang, W., Fan, J.: Mechanisms of sound scattering and radiation of submerged elastic structure-vibro-acoustic coupling of structure and water. Acta Acust. 29, 385-392 (2004)

11. Filippi, P.J.T.: Transmission and radiation of sound by thin plates. In: Filippi, P.J.T., Habault, D., Lefebvre, J.P., Bergassoli, A. (eds.) Acoustics, Basic Physics, Theory and Methods, Chapter 8. Academic, New York (1999)

12. Thomson, W.T.: Theory of Vibration with Applications. 3rd edn. Prentice Hall, London (1988)

13. Bathe, K.J.: Finite Element Procedures. Prentice Hall, New Jersey (1996)

14. Zienkiewicz, O.C., Taylor, R.L.: The Finite Element Method, 4th edn, vol. 1 (1989), vol. 2 (1991). McGraw-Hill, New York

15. Morand, H.J.P., Ohayon, R.: Fluid Structure Interaction, Applied Numerical Methods. Wiley, Chichester (1995)

16. Xing, J.T., Price, W.G.: A mixed finite element method for the dynamics analysis of coupled fluid-solid interaction problems. Proc. R. Soc. Lond. A 433, 235-255 (1991)

17. Xing, J.T., Price, W.G., Du, Q.H.: Mixed finite element substructure-subdomain methods for the dynamics analysis of coupled fluid-solid interaction problems. Phil. Trans. R. Soc. Lond. A 354, 259-295 (1996)

18. Xing, J.T., Price, W.G., Pomfred, M.J., Yan, L.H.: Natural vibration of a beam-water interaction system. J. S. \& Vib. 199, 491512 (1997)

19. Zhao, S.: Analytical and numerical investigations of linear and non-linear beam-water interaction systems. Phd thesis, School of Engineering Sciences, University of Southampton, UK (2005)

20. Xing, J.T.: The natural vibration of fluid-structure interaction systems subject to the Sommerfeld radiation condition. In: Proceedings of 2006 ASME Pressure Vessels \& Piping Division Conference, Vancouver, BC, PVP2006-ICPVT-11-93915 (2006)

21. Fung, Y.C.: A First Course in Continuum Mechanics. PrenticeHall, Englewood Cliffs (1977)

22. Xing, J.T., Price, W.G.: A power flow analysis based on continuum dynamics. Proc. R. Soc. Lond. A 455, 401-436 (1999)

23. Xing, J.T.: Two variational formulations for dynamics analysis of coupled fluid-solid interaction problems with linearised free surface wave considered. Acta Aero. Astro. Sinica 9, A568A571 (1988)

24. Xing, J.T., Price, W.G.: Variational principles of nonlinear fluidsolid interaction systems. Phil. Trans. R. Soc. Lond. A 355, 1063 1095 (1997)

25. Xing, J.T.: Natural vibration of two-dimensional slender structurewater interaction systems subject to Sommerfeld radiation condition. J. S. \& Vib. 308, 67-79 (2007)

26. Zhou, H., Wang, Y.: Application of complex mode method for offshore platform (in Chinese). Chin. Q. Mech. 23, 398-402 (2004)

27. Cui, Z., Tang, Y., Guo, C.: Fluid-structure interacted vibration of an element model of parallel-plate fuel assembly (in Chinese). J. Vib. Shock 26, 48-71 (2007) 